\title{
Triglyceride Glucose-Body Mass Index is a Reliable Indicator of Bone Mineral Density and Risk of Osteoporotic Fracture in Middle-Aged and Elderly Nondiabetic Chinese Individuals
}

\section{Zhang-Xin Wen}

the affiliated Zhuzhou Hospital of Xiangya School of Medicine of Central South University

Yong-Fang Li

National Clinical Research Center for Metabolic Diseases, the Second Xiangya Hospital of Central South University

\section{Lu-Lu Xu}

the Second Xiangya Hospital of Central South University

\section{Chun Yue}

the Second Xiangya Hospital of Central South University

\section{Qin-Yi Wang}

National Clinical Research Center for Metabolic Diseases, the Second Xiangya Hospital of Central South University

\section{Rong Chen}

the affiliated Zhuzhou Hospital of Xiangya School of Medicine of Central South University

\section{$\mathrm{Na}$ Ding}

National Clinical Research Center for Metabolic Diseases, the Second Xiangya Hospital of Central South University

\section{Xiao-Li Qu}

National Clinical Research Center for Metabolic Diseases, the Second Xiangya Hospital of Central South University

\section{Yang-Na Ou}

the Second Xiangya Hospital of Central South University

\section{Yan-Yi Yang}

the Second Xiangya Hospital of Central South University

\section{Zhi-Feng Sheng ( $\nabla$ shengzhifeng@csu.edu.cn )}

National Clinical Research Center for Metabolic Diseases, the Second Xiangya Hospital of Central South University

\section{Hong Liu}

the affiliated Zhuzhou Hospital of Xiangya School of Medicine of Central South University 


\section{Research Article}

Keywords: Triglyceride Glucose-Body Mass Index, bone mineral density, femoral neck geometry, osteoporosis, fracture, elderly

Posted Date: October 26th, 2021

DOI: https://doi.org/10.21203/rs.3.rs-1011856/v1

License: (c) (i) This work is licensed under a Creative Commons Attribution 4.0 International License.

Read Full License 


\section{Triglyceride Glucose-Body Mass Index is a Reliable}

Indicator of Bone Mineral Density and Risk of Osteoporotic Fracture in Middle-Aged and Elderly Nondiabetic Chinese

\section{Individuals}

Zhang-Xin Wen ${ }^{1,2}$, Yong-Fang $\mathrm{Li}^{1}$, Lu-Lu Xu ${ }^{3}$, Chun Yue ${ }^{3}$, Qin-Yi Wang ${ }^{1}$, Rong Chen ${ }^{1,2}$, Na Ding ${ }^{1}$, Xiao-Li Qu' ${ }^{1}$, Yang-Na Ou${ }^{4}$, Yan-Yi Yang ${ }^{3}$, Zhi-Feng Sheng ${ }^{1 *}$, Hong Liu ${ }^{2 *}$

1.Health Management Center; National Clinical Research Center for Metabolic Diseases; Department of Metabolism and Endocrinology; Hunan Provincial Key Laboratory for Metabolic Bone Diseases; the Second Xiangya Hospital of Central South University, 139 Middle Renmin Road, Changsha, 410011, Hunan, China

2.Department of Metabolism and Endocrinology, the affiliated Zhuzhou Hospital of Xiangya School of Medicine of Central South University, 116 Changjiang South Road, Zhuzhou 412007, Hunan, China

3.Health Management Center; the Second Xiangya Hospital of Central South University, 139 Middle Renmin Road, Changsha, 410011, Hunan, China

4.Hospital infection control center; the Second Xiangya Hospital of Central South University, 139 Middle Renmin Road, Changsha, 410011, Hunan, China

\section{* Correspondence:}

[Corresponding author]: Zhi-Feng Sheng, PhD

[E-mail]: shengzhifeng@ csu.edu.cn

[Corresponding author]: Hong Liu, MD

[E-mail]: 1h18607412098@163.com

Keywords: Triglyceride Glucose-Body Mass Index; bone mineral density; femoral neck geometry; osteoporosis; fracture; elderly 


\section{ABSTRACT}

Purpose: This study aimed to investigate the relationship of triglyceride glucosebody mass index (TyG-BMI) with bone mineral density, femoral neck geometry, and risk of fracture in middle-aged and elderly Chinese individuals.

Methods: A total of 832 nondiabetic individuals ( 474 men aged $\geq 50$ years and 358 postmenopausal women) were selected from the prospective population-based HOPE cohort. All individuals underwent dual-energy X-ray absorptiometry for assessment of bone mineral density (BMD) at the lumbar spine, femoral neck, and total hip, as well as femoral neck geometry. The 10-year probabilities of major osteoporotic fractures (MOFs) and hip fractures (HFs) were calculated. Correlations of TyG-BMI with BMD at different sites, femoral neck geometry, and risk of fractures were examined in men and women.

Results: Cortical thickness (CT), compression strength index (CSI), crosssectional moment of inertia (CSMI), cross-sectional area (CSA), section modulus (SM), and 25(OH)D levels were significantly lower in women (all $\mathrm{P}<0.001)$. The presence of osteoporosis was related to age, BMI, BMD and femoral neck geometry, TyG-BMI index and TyG index, MOF and HF in both sex. TyG-BMI was positively correlated with BMD at femoral neck, lumber spine, and total hip and with femoral neck geometry parameters in men, Similar significant association was also present in women. In men, TyG-BMI showed significant negative correlation with HF but not with MOF. In women, TyG-BMI showed significant negative correlation with both factors only after adjusting for other variable. The various geometric indices of the femoral neck were 
significantly impaired in individuals with low TyG-BMI. the TyG-BMI index was found to be significantly associated with osteoporosis after adjusting for confounders.

Conclusion: TyG-BMI is positively associated with BMD and femoral neck geometry, and negatively associated with risk of fracture in nondiabetic middle-aged and elderly Chinese men and women. 


\section{INTRODUCTION}

Osteoporosis is a systemic skeletal disease characterized by low bone mass and microarchitectural deterioration of bone tissue, with resulting increased bone fragility and high risk of fracture [1]. It is a major contributor to the global burden of disease, and early recognition and management will benefit both individuals and society. Bone mineral density (BMD), determined by dual-energy X-ray absorptiometry (DXA), is currently widely used to assess the quality of bone. However, only $50 \%-70 \%$ of total bone strength can be attributed to BMD. Previous research has shown that some patients with type 2 diabetes mellitus (T2DM) and obesity have high fracture risk despite having high $\mathrm{BMD}[2,3][4,5]$. Independent of $\mathrm{BMD}$, bone geometry contribute to fracture risk[6-8]. Geometric parameters of the femoral neck, such as cross-sectional area (CSA). buckling ratio (BR) and section modulus (SM) [9] also describe bone strength and are independently predictive of hip fragility fracture [10-12].

Insulin resistance (IR) does not seem to have a detrimental effect on bone mass, the most important parameter for the diagnosis of osteoporosis [13,14]. Animal research suggest that insulin exerts an anabolic effect on bone and is a critical regulator of skeletal development and structural integrity $[15,16]$. In the Rotterdam Study, which enrolled nearly 6000 elderly men and women, higher glucose and insulin levels were associated with higher bone mass at all skeletal sites, supporting the association between increased insulin levels and high BMD ${ }^{[17]}$. Abrahamsen and colleagues ${ }^{[18]}$,however, found no association between BMD and insulin, but this may have been due to the relatively small population studied. In addition to BMD, femoral neck 
geometry affects bone strength. Studies have reported an inverse association between femoral neck strength and $\mathrm{IR}^{[19-21]}$, but the study populations included individuals with and without diabetes. Shanbhogue et $\mathrm{al}^{[22]}$ reported that hyperinsulinemia directly affects bone structure, independent of obesity, in non-diabetic postmenopausal women. A few studied have examined the relationship between insulin resistance and osteoporosis in non-diabetic patients. Francisco et $\mathrm{a}^{[23]}$ conducted an research in nondiabetic postmenopausal women showed that there is a direct relationship between IR and BMD, but no association between IR and the prevalence of osteoporosis. It is possible that the relationship between IR and bone metabolism varies with sex, race, and bone mass or structure.

IR was earlier evaluated by the pancreatic suppression test, the hyperinsulinemic euglycemic clamp technique (HEGC), or the minimal model approximation of the metabolism of glucose ${ }^{[24-26]}$. but these methods are invasive, complicated, expensive, and difficult to use in the clinical setting. Meanwhile, the homeostasis model assessment-insulin resistance (HOMA-IR), which is mostly used nowadays, is limited by the absence of consensus on the reference value. Recently, the triglyceride and glucose-body mass index (TyG-BMI) — which incorporates fasting blood glucose, serum triglyceride levels, and body mass index (BMI) — has been proposed as a reliable and highly sensitive and specific alternative marker of IR ${ }^{[27,28]}$. Several studies have shown that high TyG-BMI is associated with cardiovascular events and incident nonalcoholic fatty liver disease ${ }^{[29,30]}$, and Khamseh et al. found that TyG-BMI is a reliable discriminator of liver fibrosis ${ }^{[31]}$. However, to date, no clinical studies have 
examined the association between TyG-BMI and bone metabolism. This study aimed to investigate how TyG-BMI is related to BMD, femoral neck geometry, and risk of fracture in nondiabetic Chinese middle-aged and elderly individuals.

\section{PATIENTS AND METHODS}

The study population was selected from among the participants of the HOPE study, an ongoing prospective study that is enrolling individuals undergoing physical examination at the Health Management Center of Xiangya Second Hospital. The HOPE study, which aims to achieve a sample size of 5,000 over a period of 1 year, has already accumulated more than 1,800 patients. Patients are eligible for enrollment in the HOPE study if they 1) are $\geq 40$ years old and 2) undergo DXA for BMD measurement. The exclusion criteria are 1) history of hip joint replacement or lumbar spine surgery; 2) inability to undergo DXA for any reason; 3) history of treatment with antiosteoporosis drugs; or 4) history of malignant tumor.

For the present study, we selected 832 healthy postmenopausal women and men aged $\geq 50$ years from the HOPE cohort. We excluded patients with diabetes mellitus and individuals without data on fasting blood glucose or serum triglycerides. The medical records of the selected patients were searched to obtain details such as age, years since menopause, investigation results, height, and weight. BMI was calculated as the ratio of weight (in kilograms) and height (in meters) squared $\left(\mathrm{kg} / \mathrm{m}^{2}\right)$. This study was approved by the Ethics Committee of Xiangya Second Hospital, South China University, Changsha, China (approved number LYF2021015).

DXA scans were performed by an experienced physician using the Discovery Wi 
bone densitometer (S/N87556; Hologic, USA). The regions scanned were the left femoral neck, total hip, and lumbar spine. Seven hip geometric parameters were calculated: outer diameter, CSA, cortical thickness (CT), cross-sectional moment of inertia (CSMI), compression strength index (CSI), SM and BR (from BMD), and areal bone size data. Outer diameter refers to the outer diameter of the femoral neck at its midpoint. Endocortical diameter refers to the endocortical diameter of the femoral neck at the midpoint. CSA is an index of axial compression strength and reflects the resistance to loads directed along the bone axis. CSMI is a measure of the mass distribution relative to the geometric center; it reflects how effective a cross-section is at resisting bending and torsion-depending on the axis chosen for calculation. CT is an estimate of mean cortical thickness. BR is an index of bone geometric instability and reflects the resistance against compressive stress, which could lead to sudden sideways deflection of the structural member; higher BR values indicate greater instability and higher fracture risk ${ }^{[32-34]}$. CSI is a measure of the ability of the femoral neck to withstand compressive load in the axial dimension. SM, computed as CSMI divided by the distance from the bone edge to the centroid, describes femoral neck bending strength. A China-specific fracture risk assessment tool (FRAX) algorithm (which included the femoral neck BMD T-score) was used to determine the 10-year probability of major osteoporotic fractures (MOFs) and hip fractures (HFs).

Blood samples were obtained after an overnight fast of at least 8 hours. Fasting blood glucose, serum triglycerides, serum total cholesterol, and serum high-density lipoprotein cholesterol were measured using an ADVIA 1650 Chemistry Analyzer 
(Siemens, Washington, DC, USA) in 2007, and a Hitachi 7600 Automatic Analyzer

(Hitachi, Tokyo, Japan) from 2008 to 2010. Serum levels of total 25-hydroxyvitamin D (25OHD) was measured by an automated chemiluminescence system. TyG-BMI was calculated using the formula ${ }^{[35]}$. TyG-BMI $=\operatorname{Ln}[$ fasting glucose $(\mathrm{mg} / \mathrm{dL}) \times$ triglycerides $(\mathrm{mg} / \mathrm{dL}) / 2] \times \mathrm{BMI}$.

\section{Statistical Analysis}

Continuous data were assessed for normality and analyzed using the independent samples $t$-test or the Mann-Whitney $U$ test, as appropriate. Pearson or Spearman correlation was used to examine associations between TyG-BMI and BMD, femoral neck geometry, and risk of fracture. Participants were stratified by sex to examine sexspecific associations. Multivariable linear regression analysis was used to explore associations between TyG-BMI and femoral neck parameters (bone density and femoral neck geometry) in the two sexes. Multivariable logistic regression analysis was used to explore associations between TyG-BMI and osteoporosis. All analyses were performed using SPSS 26 (IBM Corp., Armonk, NY, USA). Statistical significance was at $\mathrm{P}<0.05$.

\section{RESULTS}

\section{Clinical Characteristics of the Study Population}

Characteristics of the 832 individuals (474 men, 358 women) are summarized in Table S1 and Table1. Mean age was comparable between men and women (59.0 \pm 7.95 years vs. $59.6 \pm 7.72$ years, $\mathrm{P}=0.126$ ). Mean TyG-BMI was significantly higher in men than in women $(219.6 \pm 32.5$ vs. $202.5 \pm 29.8, \mathrm{P}<0.001)$. The prevalence of osteoporosis (as diagnosed by the BMD T-score) was higher in women than in men 
$(20.1 \%$ vs. $9.5 \%, \mathrm{p}<0.05)$. Other detailed baseline characteristics are presented in

Additional file 1: Table S1. Compared to non-osteoporotic group, the presence of osteoporosis was related to age, BMI, BMD and femoral neck geometry, TyG-BMI index and TyG index, MOF and HF in both sex (Table 1).

\section{Association of TyG-BMI with BMD}

Figure 1 shows the correlation of TyG-BMI with BMD at different sites. In men, TyG-BMI was positively correlated with femoral neck-BMD $(\mathrm{r}=0.236, \mathrm{P}<0.001)$, total hip-BMD ( $\mathrm{r}=0.249, \mathrm{P}<0.001)$, and lumbar spine-BMD $(\mathrm{r}=0.145, \mathrm{P}=0.002)$. Similarly, in women, TyG-BMI index was positively correlated with femoral neckBMD $(r=0.186, P<0.001)$, total hip-BMD $(r=0.259, \mathrm{P}<0.001)$, and lumbar spine$\mathrm{BMD}(\mathrm{r}=0.133, \mathrm{P}=0.013)$. In both sexes, the association persisted even after adjusting for age, smoking, drinking, and history of previous hip fracture or parental hip fracture (Table 3).

\section{Association of TyG-BMI with Femoral Neck Geometry and Risk of Fracture}

In unadjusted analysis (Table 2), TyG-BMI was positively correlated with femoral neck CSA, CSMI, SM, and CT in both men and women (all $\mathrm{P}<0.001$ ), but negatively correlated with BR and CSI. In both sexes, the associations remained statistically significant even after adjusting for age, smoking, drinking, and history of previous hip fracture or parental hip fracture (Table 3). In men, TyG-BMI was significantly correlated to HF but not to MOF; in women, TyG-BMI was not significantly correlated with either of the two factors $(\mathrm{P}>0.05)$. However, in women, after adjusting for age, smoking, drinking, and history of previous hip fracture and parental hip fracture, TyG- 
BMI was significantly associated with MOF and HF; in men, the TyG-BMI remained significantly associated with HF.

Multiple Linear Regression Analysis of Association between TyG-BMI and BMD and Bone Geometric Parameters

Linear regression analysis showed that TyG-BMI was a significant independent predictor of BMD, SM, CSA, CSI, and BR. The standardized regression coefficients of TyG-BMI in men(Table 3a) and women(Table 3b), respectively, were $0.234(\mathrm{P}<0.001)$ and $0.279(\mathrm{P}<0.001)$ for femoral neck BMD, $0.176(\mathrm{P}<0.001)$ and $0.192(\mathrm{P}<0.001)$ for total hip BMD, $0.238(\mathrm{P}<0.001)$ and $0.283(\mathrm{P}<0.001)$ for CSA, $0.212(\mathrm{P}<0.001)$ and $0.228(\mathrm{p}<0.001)$ for $\mathrm{SM}$, and $-0.202(\mathrm{P}<0.001)$ and $0.282(\mathrm{P}<0.001)$ for $\mathrm{BR}$.

\section{Distributions of the TyG-BMI index according to the bone health status.}

In the evaluation of the relationship between TyG-BMI index and the bone health status, patients with osteoporosis had a lower TyG-BMI index $(\mathrm{P}$ for trend $=0.006)$ compared to the others, showing a dose-response manner (Figure. 2).

\section{Multivariable logistic regression analyses between possible predictors and} osteoporosis.

In the multivariable logistic regression analyses, the TyG-BMI index was found to be significantly associated with osteoporosis (adjusted odds ratio $[\mathrm{aOR}]=1.019 ; 95 \%$ confidence interval $[\mathrm{CI}]=1.01-1.028)$ after adjusting for confounders. Age (aOR $=0.919 ; 95 \% \mathrm{CI}=0.892-0.947)$ and $\mathrm{Sex}$, female $(\mathrm{aOR}=0.489 ; 95 \% \mathrm{CI}=0.266-0.889)$ were also related to osteoporosis (Table 4).

\section{DISCUSSION}


This is the first study to investigate the association of TyG-BMI with bone mass and femoral neck geometry in healthy, nondiabetic, middle-age and elderly individuals. The proximal femur, hip, and the lumbar spine were examined, as these are anatomical sites at high risk of osteoporotic fractures. As measures of bone strength, we used areal BMD (aBMD) and femoral geometry. TyG-BMI, which combines serum triglycerides, fasting plasma glucose, and obesity status, is considered more reliable than TyG for the identification of IR. It is a less expensive and more reliable marker of IR than traditional markers like HOMA-IR ${ }^{[36,37]}$.

We demonstrated that higher aBMD was associated with higher bone strength and lower fracture risk at all sites, with the association being significantly stronger in men than in postmenopausal women. The sex differences in these parameters may explain the higher incidence of fragility fractures in women. It also implies that sex-dependent femoral neck geometry contributes significantly to the ability to withstand stress.

The relationship between IR and BMD has been studied in different populations but the results have been mixed. Consistent with Riggs et al. [38]. we found that higher TyG-BMI was associated with greater aBMD at both weight-bearing and non-weightbearing skeletal sites, TyG-BMI index was significantly associated with osteoporosis. Previous studies have reported association between bone metabolism and HOMA-IR, another surrogate marker of IR. Further, greater IR was found to be associated with higher BMD ${ }^{[39,40]}$. In a study of Caucasian non-diabetic women from the Study of Women's Health Across the Nation (SWAN) found that higher IR is associated with greater volumetric BMD and generally favorable bone microarchitecture at non- 
weight-bearing distal radius and weight-bearing distal tibia, independent of body weight ${ }^{[20]}$. This effect of hyperinsulinism on BMD may be because insulin exerts peripheral osteogenic effects via stimulation of osteoblasts or inhibition of osteoclasts. All these research results suggest that TyG-BMI index is a protective factor for osteoporosis. However. Shin et al ${ }^{[41]}$ reported an inverse relationship between HOMAIR and aBMD in a population-based study of young South Korean men (mean age, 49.9 years) suggesting that IR is a negative predictor of bone health. Our study population differs from that of Shin et al. ${ }^{[41]}$ in several aspects: our study participants were Chinese, older (mean age, 60.3 years) and, importantly, nondiabetic. It is currently unknown whether the effects of IR or hyperinsulinemia on bone are age-, sex- or race-specific. The inclusion of patients with diabetes in Shin et al. ${ }^{[41]}$ may have confounded their results as HOMA-IR is unreliable in patients on antidiabetic medications; further, chronic hyperglycemia and/or antidiabetic medications may affect skeletal microarchitecture. Thus, comparison of their findings with ours is difficult.

In this study we also investigated the association between TyG-BMI and femoral neck geometry. A recent study showed that several conditions associated with altered bone metabolism (for example, sarcopenia) result in poor femoral neck geometry, suggesting that these indices on DXA scans may be a good indicator of bone health ${ }^{[42]}$. Consistent with a previous Chinese study ${ }^{[43]}$, we found that CSA, CT, SM, CSMI, and CSI decrease with age, whereas BR increases with age. These results imply the decrease in CSA, CT, SM, CSMI, and CSI and increase in BR might contribute to fragility fractures of the femoral neck in old age. Further, CSA, CT, SM, CSMI, and CSI were 
lower, and BR higher, in women than in men, which may explain the greater vulnerability of the femoral neck in the former.

There is a paucity of data describing the relationships between IR and femoral neck geometry. In the present study, TyG-BMI was positively associated with femoral neck CSA and SM, but negatively associated with BR; the relationships remained statistically significant even after controlling for age and previous fracture, suggesting that IR contributed to favorable femoral neck geometry. A possible explanation for this relationship is that insulin has an anabolic effect on bone, stimulating osteoblast growth and proliferation on periosteal surfaces and thus increasing SM and CSA. Overall, the results suggest that TyG-BMI has a positive effect on bone geometry in middle-aged and elderly Chinese individuals. Contrary to our findings, an inverse association between IR and bone size has been demonstrated in nondiabetic postmenopausal Caucasian women ${ }^{[22]}$.In Korean men and women, with and without diabetes, HOMA-IR and fasting insulin levels were found to be inversely associated with composite indices of femoral neck strength ${ }^{[44]}$. The primary difference between our study and the studies mentioned above is in the populations enrolled, implying that differences in sex, age, diabetes status, and race affect the relationship between IR and bone structure.

This study has some limitations. First, this was a retrospective study, so we cannot infer that IR leads to high bone strength; that will have to be clarified in longitudinal studies. Second, serum levels of hormones that affect bone metabolism (e.g., estrogen, androgen, and pituitary gonadotropin) were not evaluated in our study. Third, we did 
not use the HEGC technique and HOMA-IR for measuring IR, these methods, although accurate and popular, are time-consuming and costly and therefore not suitable for application in large samples. TyG-BMI is a reliable and highly sensitive alternative to $\mathrm{HEGC}^{[27]}$.

In summary, TyG-BMI may be a reliable indicator of favorable bone density and strength in healthy, nondiabetic postmenopausal women and men and could be useful in the clinic to evaluate and predict the risk of osteoporosis. More mechanistic studies, both preclinical and clinical, are needed to better understand the effect of IR on bone and to clarify whether insulin resistance-related changes affect fracture risk.

\section{ABBREVIATIONS:}

AGE, glycation end product

BMD, bone mineral density

BMI, body mass index

$\mathrm{BR}$, buckling ratio

CSA, cross-sectional area

CSI, compression strength index

CSMI, cross-sectional moment of inertia

CT, cortical thickness

DXA, dual-energy X-ray absorptiometry

HEGC technique, hyperinsulinemic euglycemic clamp technique

HFs, hip fractures (HFs)

HOMA-IR, homeostatic model assessment for insulin resistance 
IR, insulin resistance

MOFs, major osteoporotic fractures

SM, section modulus

T2DM, type 2 diabetes mellitus

TyG-BMI index, triglyceride and glucose-body mass index

\section{Declarations :}

\section{Consent for publication}

Not applicable

\section{Availability of data and materials}

The datasets used and/or analysed during the current study are available from the corresponding author on reasonable request.

\section{Competing interests}

The authors declare that the research was conducted in the absence of any commercial or financial relationships that could be construed as a potential conflict of interest.

\section{Funding}

This work was supported by grants from the National Nature Science Foundation of China [grant numbers 81870622], the Hunan Nature Science Foundation [grant number 2018JJ2574], the Changsha Nature Science Foundation [grant number kq2014251] and Bethune Charitable Foundation, BCF [grant number G-X-2019-1107-3] 


\section{Author Contributions}

The study conception and design were performed by SF and LH. Data collection and analysis were performed by WZ, XL, LY, YY, CR, WQ. Data verification and the first draft of the manuscript was written by WZ, DN, QX, YC. OY and CR were responsible for statistical analysis. All authors approved the final manuscript and taking final responsibility for the paper.

\section{Acknowledgments}

Not applicable

\section{Reference}

1. Kado D, Browner W, Blackwell T, Gore R, Cummings SR. Rate of bone loss is associated with mortality in older women: a prospective study. J Bone Miner Res (2000) 15(10):1974-80. doi: 10.1359/jbmr.2000.15.10.1974.

2. Bonds D, Larson J, Schwartz A, Strotmeyer ES, Robbins J, Rodriguez BL, et al. Risk of fracture in women with type 2 diabetes: the Women's Health Initiative Observational Study. J Clin Endocrinol Metab (2006) 91(9):3404-3410. doi: 10.1210/jc.2006-0614.

3. Vestergaard PJ. Discrepancies in bone mineral density and fracture risk in patients with type 1 and type 2 diabetes - a meta-analysis. Osteoporos Int (2007) 18(4):427-444. doi: 10.1007/s00198-006-0253-4

4. Siris ES, Chen Y-T, Abbott TA, Barrett-Connor E, Miller PD, Wehren LE, et al. Bone mineral density thresholds for pharmacological intervention to prevent fractures. Arch Intern Med (2004) 164(10):1108-12. doi: 10.1001/archinte.164.10.1108 
5. Stone KL, Seeley DG, Lui L-Y, Cauley JA, Ensrud K, Browner WS, et al. BMD at multiple sites and risk of fracture of multiple types: long-term results from the Study of Osteoporotic Fractures. J Bone Miner Res (2003) 18(11):1947-54. doi: 10.1359/jbmr.2003.18.11.1947.

6. Alonso CG, Curiel MD, Carranza FH, Cano RP, Peréz AD, et al. Femoral bone mineral density, neck-shaft angle and mean femoral neck width as predictors of hip fracture in men and women. Multicenter Project for Research in Osteoporosis. Osteoporos Int. 2000;11(8):714-720. doi: 10.1007/s001980070071.

7. De Laet C, Kanis JA, Odén A, Johanson H, Johnell O, Delmas P, et al . Body mass index as a predictor of fracture risk: a meta-analysis. Osteoporos Int. 2005 Nov;16(11):1330-8. doi: 10.1007/s00198-005-1863-y

8. Pulkkinen P, Partanen J, Jalovaara P, Jämsä T. Combination of bone mineral density and upper femur geometry improves the prediction of hip fracture. Osteoporos Int. 2004 Apr;15(4):274-80. doi: 10.1007/s00198-003-1556-3.

9. Black DM, Bouxsein ML, Marshall LM, Cummings SR, Lang TF, Cauley JA, et al. Osteoporotic Fractures in Men (MrOS) Research Group. Proximal femoral structure and the prediction of hip fracture in men: a large prospective study using QCT. J Bone Miner Res.2008 Aug;23(8):1326-33.doi: 10.1359/jbmr.080316.

10. Danielson ME, Beck TJ, Lian Y, Karlamangla AS, Greendale GA, Ruppert K, et al . Ethnic variability in bone geometry as assessed by hip structure analysis: 
findings from the hip strength across the menopausal transition study. J Bone Miner Res. 2013 Apr;28(4):771-9. doi: 10.1002/jbmr.1781.

11. Khoo BC, Lewis JR, Brown K, Prince RL. Evaluation of a simplified hip structure analysis method for the prediction of incident hip fracture events. Osteoporos Int. 2016 Jan;27(1):241-8. doi: 10.1007/s00198-015-3282-z.

12. Litwic AE, Clynes M, Denison HJ, Jameson KA, Edwards MH, Sayer AA, et al . Non-invasive Assessment of Lower Limb Geometry and Strength Using Hip Structural Analysis and Peripheral Quantitative Computed Tomography: A Population-Based Comparison. Calcif Tissue Int. 2016 Feb;98(2):158-64. doi: $10.1007 / \mathrm{s} 00223-015-0081-7$.

13. Conte C, Epstein S, Napoli N. Insulin resistance and bone: a biological partnership. Acta Diabetol. 2018 Apr;55(4):305-314. doi: 10.1007/s00592-0181101-7.

14. de Paula FJ, de Araújo IM, Carvalho AL, Elias J Jr, Salmon CE, NogueiraBarbosa MH. The Relationship of Fat Distribution and Insulin Resistance with Lumbar Spine Bone Mass in Women. PLoS One. 2015 Jun 11;10(6): e0129764. doi: 10.1371/journal.pone.0129764.

15. Nyman JS, Kalaitzoglou E, Clay Bunn R, Uppuganti S, Thrailkill KM, Fowlkes JL. Preserving and restoring bone with continuous insulin infusion therapy in a mouse model of type 1 diabetes. Bone Rep. 2017 Jul 4;7:1-8. doi: 10.1016/j.bonr.2017.07.001.

16. Thrailkill K, Bunn RC, Lumpkin C Jr, Wahl E, Cockrell G, Morris L, et al . Loss 
of insulin receptor in osteoprogenitor cells impairs structural strength of bone. J Diabetes Res. 2014;2014:703589. doi: 10.1155/2014/703589.

17. Stolk RP, Van Daele PL, Pols HA, Burger H, Hofman A, Birkenhäger JC, et al . Hyperinsulinemia and bone mineral density in an elderly population: The Rotterdam Study. Bone. 1996 Jun;18(6):545-9. doi: 10.1016/87563282(96)00079-8.

18. Abrahamsen B, Rohold A, Henriksen JE, Beck-Nielsen H. Correlations between insulin sensitivity and bone mineral density in non-diabetic men. Diabet Med. 2000 Feb;17(2):124-9. doi: 10.1046/j.1464-5491.2000.00234.x.

19. Srikanthan P, Crandall CJ, Miller-Martinez D, Seeman TE, Greendale GA, Binkley N, et al. Insulin resistance and bone strength: findings from the study of midlife in the United States. J Bone Miner Res. 2014 Apr;29(4):796-803. doi: 10.1002/jbmr.2083.

20. Shanbhogue VV, Finkelstein JS, Bouxsein ML, Yu EW. Association Between Insulin Resistance and Bone Structure in Nondiabetic Postmenopausal Women. J Clin Endocrinol Metab. 2016 Aug;101(8):3114-22. doi: 10.1210/jc.2016-1726

21. Srikanthan P, Crandall CJ, Miller-Martinez D, Seeman TE, Greendale GA, Binkley N, et al. Insulin resistance and bone strength: findings from the study of midlife in the United States. J Bone Miner Res. 2014 Apr;29(4):796-803. doi: 10.1002/jbmr.2083.

23. Shanbhogue VV, Finkelstein JS, Bouxsein ML, Yu EW. Association Between Insulin Resistance and Bone Structure in Nondiabetic Postmenopausal Women. 
J Clin Endocrinol Metab. 2016 Aug;101(8):3114-22. doi: 10.1210/jc.2016-1726

23. Campillo-Sánchez F, Usategui-Martín R, Ruiz-de Temiño Á, Gil J, RuizMambrilla M, Fernández-Gómez JM, et al. Relationship between Insulin Resistance (HOMA-IR), Trabecular Bone Score (TBS), and Three-Dimensional Dual-Energy X-ray Absorptiometry (3D-DXA) in Non-Diabetic Postmenopausal Women. J Clin Med 2020;9(6). doi: 10.3390/jcm9061732.

24. DeFronzo RA, Tobin JD, Andres R. Glucose clamp technique: a method for quantifying insulin secretion and resistance. Am J Physiol. 1979 Sep;237(3): E214-23. doi: 10.1152/ajpendo.1979.237.3. E214.

25. Bergman RN, Prager R, Volund A, Olefsky JM. Equivalence of the insulin sensitivity index in man derived by the minimal model method and the euglycemic glucose clamp. J Clin Invest. 1987 Mar;79(3):790-800. doi: 10.1172/JCI112886.

26. Greenfield MS, Doberne L, Kraemer F, Tobey T, Reaven G. Assessment of insulin resistance with the insulin suppression test and the euglycemic clamp. Diabetes. 1981 May;30(5):387-92. doi: 10.2337/diab.30.5.387.

27. Guerrero-Romero F, Simental-Mendía LE, González-Ortiz M, MartínezAbundis E, Ramos-Zavala MG, Hernández-González SO, et al . The product of triglycerides and glucose, a simple measure of insulin sensitivity. Comparison with the euglycemic-hyperinsulinemic clamp. J Clin Endocrinol Metab. 2010 Jul;95(7):3347-51. doi: 10.1210/jc.2010-0288.

28. Lee J, Kim B, Kim W, Ahn C, Choi HY, Kim JG, et al. Lipid indices as simple 
and clinically useful surrogate markers for insulin resistance in the U.S. population. Sci Rep. 2021 Jan 27;11(1):2366. doi: 10.1038/s41598-021-820532.

29. Ma X, Dong L, Shao Q, Cheng Y, Lv S, Sun Y, et al. Triglyceride glucose index for predicting cardiovascular outcomes after percutaneous coronary intervention in patients with type 2 diabetes mellitus and acute coronary syndrome. Cardiovasc Diabetol. 2020 Mar 10;19(1):31. doi: 10.1186/s12933020-01006-7.

30. Kitae A, Hashimoto Y, Hamaguchi M, Obora A, Kojima T, Fukui M. The Triglyceride and Glucose Index Is a Predictor of Incident Nonalcoholic Fatty Liver Disease: A Population-Based Cohort Study. Can J Gastroenterol Hepatol. 2019 Oct 7; 2019:5121574. doi: 10.1155/2019/5121574.

31. Khamseh ME, Malek M, Abbasi R, Taheri H, Lahouti M, Alaei-Shahmiri F. Triglyceride Glucose Index and Related Parameters (Triglyceride GlucoseBody Mass Index and Triglyceride Glucose-Waist Circumference) Identify Nonalcoholic Fatty Liver and Liver Fibrosis in Individuals with Overweight/Obesity. Metab Syndr Relat Disord. 2021 Apr;19(3):167-173. doi: 10.1089/met.2020.0109.

32. Anitha D, Lee T. Assessing bone quality in terms of bone mineral density, buckling ratio and critical fracture load. J Bone Metab. 2014 Nov;21(4):243-7. doi: 10.11005/jbm.2014.21.4.243.

33. Sheu Y, Cauley JA, Patrick AL, Wheeler VW, Bunker CH, Zmuda JM. Risk 
factors for fracture in middle-age and older-age men of African descent. J Bone Miner Res. 2014 Jan;29(1):234-41. doi: 10.1002/jbmr.2016.

34. Duan Y, Beck TJ, Wang XF, Seeman E. Structural and biomechanical basis of sexual dimorphism in femoral neck fragility has its origins in growth and aging. J Bone Miner Res. 2003 Oct;18(10):1766-74. doi: 10.1359/jbmr.2003.18.10.1766.

35. Er LK, Wu S, Chou HH, Hsu LA, Teng MS, Sun YC, et al. Triglyceride Glucose-Body Mass Index Is a Simple and Clinically Useful Surrogate Marker for Insulin Resistance in Nondiabetic Individuals. PLoS One. 2016 Mar 1;11(3): e0149731. doi: 10.1371/journal.pone.0149731.

36. Locateli JC, Lopes WA, Simões CF, de Oliveira GH, Oltramari K, Bim RH, et al. Triglyceride/glucose index is a reliable alternative marker for insulin resistance in South American overweight and obese children and adolescents. J Pediatr Endocrinol Metab. 2019 Oct 25;32(10):1163-1170. doi: 10.1515/jpem2019-0037.

37. Guerrero-Romero F, Simental-Mendía LE, González-Ortiz M, MartínezAbundis E, Ramos-Zavala MG, Hernández-González SO, et al. The product of triglycerides and glucose, a simple measure of insulin sensitivity. Comparison with the euglycemic-hyperinsulinemic clamp. J Clin Endocrinol Metab. 2010 Jul;95(7):3347-51. doi: 10.1210/jc.2010-0288.

38. Riggs BL, Melton Iii LJ 3rd, Robb RA, Camp JJ, Atkinson EJ, Peterson JM, et al. Population-based study of age and sex differences in bone volumetric density, 
size, geometry, and structure at different skeletal sites. J Bone Miner Res. 2004 Dec;19(12):1945-54. doi: 10.1359/JBMR.040916.

39. Kalimeri M, Leek F, Wang NX, Koh HR, Roy NC, Cameron-Smith D, et al. Association of Insulin Resistance with Bone Strength and Bone Turnover in Menopausal Chinese-Singaporean Women without Diabetes. Int J Environ Res Public Health. 2018 Apr 30;15(5):889. doi: 10.3390/ijerph15050889.

40. Yoon JH, Hong AR, Choi W, Park JY, Kim HK, Kang HC. Association of Triglyceride-Glucose Index with Bone Mineral Density in Non-diabetic Koreans: KNHANES 2008-2011. Calcif Tissue Int. 2021 Feb;108(2):176-187. doi: 10.1007/s00223-020-00761-9.

41. Shin D, Kim S, Kim KH, Lee K, Park SM. Association between insulin resistance and bone mass in men. J Clin Endocrinol Metab. 2014 Mar;99(3):988-95. doi: 10.1210/jc.2013-3338.

42. Saddik H, Nasr R, Pinti A, Watelain E, Fayad I, Baddoura R, et al. Sarcopenia negatively affects hip structure analysis variables in a group of Lebanese postmenopausal women. BMC Bioinformatics. 2020 Mar 11;21(Suppl 2):86. doi: 10.1186/s12859-020-3353-9.

43. Shen Y, Tang ML, Wu XP, Yuan LQ, Dai RC, Zhang H, et al. Gender differences in a reference database of age-related femoral neck geometric parameters for Chinese population and their association with femoral neck fractures. Bone. 2016 Dec; 93:64-70. doi: 10.1016/j.bone.2016.09.014.

44. Ahn SH, Kim H, Kim B-J, Lee, SH, Koh, JM ,et al. Insulin resistance and 
composite indices of femoral neck strength in Asians: the fourth Korea National Health and Nutrition Examination Survey (KNHANES IV). Clinical Endocrinology. 2016;84(2):185-193. doi: 10.1111/cen.12958 
[1] Kado D, Browner W, Blackwell T, et al. Rate of bone loss is associated with mortality in older women: a prospective study. 2000;15(10):1974-1980.

[2] Bonds D, Larson J, Schwartz A, et al. Risk of fracture in women with type 2 diabetes: the Women's Health Initiative Observational Study. 2006;91(9):3404-3410.

[3] Vestergaard PJOiajearocbtEFfO, USA tNOFot. Discrepancies in bone mineral density and fracture risk in patients with type 1 and type 2 diabetes--a meta-analysis. 2007;18(4):427444.

[4] Siris E, Chen Y, Abbott T, et al. Bone mineral density thresholds for pharmacological intervention to prevent fractures. 2004;164(10):1108-1112.

[5] Stone KL, Seeley DG, Lui LY, et al. BMD at multiple sites and risk of fracture of multiple types: Long-term results from the study of osteoporotic fractures. Journal of Bone and Mineral Research. 2003;18(11):1947-1954.

[6] Alonso C, Curiel M, Carranza F, et al. Femoral bone mineral density, neck-shaft angle and mean femoral neck width as predictors of hip fracture in men and women. Multicenter Project for Research in Osteoporosis. 2000;11(8):714-720.

[7] De Laet C, Kanis J, Odén A, et al. Body mass index as a predictor of fracture risk: a meta analysis. 2005;16(11):1330-1338.

[8] Pulkkinen P, Partanen J, Jalovaara P, et al. Combination of bone mineral density and upper femur geometry improves the prediction of hip fracture. 2004;15(4):274-280.

[9] Black D, Bouxsein M, Marshall L, et al. Proximal femoral structure and the prediction of hip fracture in men: a large prospective study using QCT. 2008;23(8):1326-1333.

[10] Danielson M, Beck T, Lian Y, et al. Ethnic variability in bone geometry as assessed by hip structure analysis: findings from the hip strength across the menopausal transition study. 2013;28(4):771-779.

[11] Khoo B, Lewis J, Brown K, et al. Evaluation of a simplified hip structure analysis method for the prediction of incident hip fracture events. 2016;27(1):241-248.

[12] Litwic A, Clynes M, Denison H, et al. Non-invasive Assessment of Lower Limb Geometry and Strength Using Hip Structural Analysis and Peripheral Quantitative Computed Tomography: A Population-Based Comparison. 2016;98(2):158-164.

[13] Conte C, Epstein S, Napoli N. Insulin resistance and bone: a biological partnership. Acta Diabetologica. 2018;55(4):305-314.

[14] de Paula FJA, de Araujo IM, Carvalho AL, et al. The Relationship of Fat Distribution and Insulin Resistance with Lumbar Spine Bone Mass in Women. Plos One. 2015;10(6).

[15] Nyman JS, Kalaitzoglou E, Clay Bunn R, et al. Preserving and restoring bone with continuous insulin infusion therapy in a mouse model of type 1 diabetes. Bone reports. 2017;7:1-8.

[16] Thrailkill K, Bunn RC, Lumpkin C, Jr., et al. Loss of Insulin Receptor in Osteoprogenitor Cells Impairs Structural Strength of Bone. Journal of Diabetes Research. 2014;2014.

[17] Stolk RP, Van Daele PL, Pols HA, et al. Hyperinsulinemia and bone mineral density in an elderly population: The Rotterdam Study. Bone. 1996;18(6):545-549.

[18] Abrahamsen B, Rohold A, Henriksen JE, et al. Correlations between insulin sensitivity and bone mineral density in non-diabetic men. Diabet Med. 2000;17(2):124-129.

[19] Srikanthan P, Crandall CJ, Miller-Martinez D, et al. Insulin Resistance and Bone Strength: Findings From the Study of Midlife in the United States. Journal of Bone and Mineral 
Research. 2014;29(4):796-803.

[20] Shanbhogue VV, Finkelstein JS, Bouxsein ML, et al. Association Between Insulin Resistance and Bone Structure in Nondiabetic Postmenopausal Women. Journal of Clinical Endocrinology \& Metabolism. 2016;101(8):3114-3122.

[21] Srikanthan P, Crandall CJ, Miller-Martinez D, et al. Insulin resistance and bone strength: findings from the study of midlife in the United States. J Bone Miner Res. 2014;29(4):796803.

[22] Shanbhogue V, Finkelstein J, Bouxsein M, et al. Association Between Insulin Resistance and Bone Structure in Nondiabetic Postmenopausal Women. 2016;101(8):3114-3122.

[23] Campillo-Sanchez F, Usategui-Martin R, Ruiz de Temino A, et al. Relationship between Insulin Resistance (HOMA-IR), Trabecular Bone Score (TBS), and Three-Dimensional Dual-Energy X-ray Absorptiometry (3D-DXA) in Non-Diabetic Postmenopausal Women. Journal of Clinical Medicine. 2020;9(6).

[24] DeFronzo R, Tobin J, Andres RJTAjop. Glucose clamp technique: a method for quantifying insulin secretion and resistance. 1979;237(3):E214-223.

[25] Bergman $R$, Prager $R$, Volund $A$, et al. Equivalence of the insulin sensitivity index in man derived by the minimal model method and the euglycemic glucose clamp. 1987;79(3):790-800.

[26] Greenfield M, Doberne L, Kraemer F, et al. Assessment of insulin resistance with the insulin suppression test and the euglycemic clamp. 1981;30(5):387-392.

[27] Guerrero-Romero F, Simental-Mendía L, González-Ortiz M, et al. The product of triglycerides and glucose, a simple measure of insulin sensitivity. Comparison with the euglycemic-hyperinsulinemic clamp. 2010;95(7):3347-3351.

[28] Lee J, Kim B, Kim W, et al. Lipid indices as simple and clinically useful surrogate markers for insulin resistance in the U.S. population. 2021;11(1):2366.

[29] Ma X, Dong L, Shao Q, et al. Triglyceride glucose index for predicting cardiovascular outcomes after percutaneous coronary intervention in patients with type 2 diabetes mellitus and acute coronary syndrome. Cardiovascular Diabetology. 2020;19(1).

[30] Kitae A, Hashimoto $Y$, Hamaguchi M, et al. The Triglyceride and Glucose Index Is a Predictor of Incident Nonalcoholic Fatty Liver Disease: A Population-Based Cohort Study. Canadian Journal of Gastroenterology and Hepatology. 2019;2019.

[31] Khamseh ME, Malek M, Abbasi R, et al. Triglyceride Glucose Index and Related Parameters (Triglyceride Glucose-Body Mass Index and Triglyceride Glucose-Waist Circumference) Identify Nonalcoholic Fatty Liver and Liver Fibrosis in Individuals with Overweight/Obesity. Metabolic Syndrome and Related Disorders. 2020.

[32] Anitha D, Lee T. Assessing bone quality in terms of bone mineral density, buckling ratio and critical fracture load. Journal of bone metabolism. 2014;21(4):243-247.

[33] Sheu Y, Cauley JA, Patrick AL, et al. Risk Factors for Fracture in Middle-Age and OlderAge Men of African Descent. Journal of Bone and Mineral Research. 2014;29(1):234-241.

[34] Duan YB, Beck TJ, Wang XF, et al. Structural and biomechanical basis of sexual dimorphism in femoral neck fragility has its origins in growth and aging. Journal of Bone and Mineral Research. 2003;18(10):1766-1774.

[35] Er L, Wu S, Chou H, et al. Triglyceride Glucose-Body Mass Index Is a Simple and Clinically Useful Surrogate Marker for Insulin Resistance in Nondiabetic Individuals. 
2016;11(3):e0149731.

[36] Locateli JC, Lopes WA, Simoes CF, et al. Triglyceride/glucose index is a reliable alternative marker for insulin resistance in South American overweight and obese children and adolescents. Journal of Pediatric Endocrinology \& Metabolism. 2019;32(10):1163-1170.

[37] Guerrero-Romero F, Simental-Mendia LE, Gonzalez-Ortiz M, et al. The Product of Triglycerides and Glucose, a Simple Measure of Insulin Sensitivity. Comparison with the Euglycemic-Hyperinsulinemic Clamp. Journal of Clinical Endocrinology \& Metabolism. 2010;95(7):3347-3351.

[38] Riggs B, Melton lii L, Robb R, et al. Population-based study of age and sex differences in bone volumetric density, size, geometry, and structure at different skeletal sites. 2004;19(12):1945-1954.

[39] Kalimeri M, Leek F, Wang N, et al. Association of Insulin Resistance with Bone Strength and Bone Turnover in Menopausal Chinese-Singaporean Women without Diabetes. 2018;15(5).

[40] Yoon JH, Hong AR, Choi W, et al. Association of Triglyceride-Glucose Index with Bone Mineral Density in Non-diabetic Koreans: KNHANES 2008-2011. Calcified Tissue International. 2021;108(2):176-187.

[41] Shin D, Kim S, Kim KH, et al. Association Between Insulin Resistance and Bone Mass in Men. Journal of Clinical Endocrinology \& Metabolism. 2014;99(3):988-995.

[42] Saddik H, Nasr R, Pinti A, et al. Sarcopenia negatively affects hip structure analysis variables in a group of Lebanese postmenopausal women. Bmc Bioinformatics. 2020;21.

[43] Shen Y, Tang M, Wu X, et al. Gender differences in a reference database of age-related femoral neck geometric parameters for Chinese population and their association with femoral neck fractures. 2016;93:64-70.

[44] Ahn SH, Kim H, Kim B-J, et al. Insulin resistance and composite indices of femoral neck strength in Asians: the fourth Korea National Health and Nutrition Examination Survey (KNHANES IV). Clinical Endocrinology. 2016;84(2):185-193. 
Table 1 Baseline characteristics of osteoporosis and non-osteoporosis group

\begin{tabular}{|c|c|c|c|c|}
\hline & Men & & Women & \\
\hline & Osteoporosis & Non-osteoporosis & Osteoporosis & Non-osteoporosis \\
\hline Age (years) & $62.0 \pm 1.00 *$ & $59.0 \pm 0.00$ & $65.0 \pm 1.00 *$ & $59.0 \pm 0.00$ \\
\hline Height $(\mathrm{cm})$ & $164.3 \pm 0.90 *$ & $167.7 \pm 0.30$ & $152.8 \pm 0.80 *$ & $156.5 \pm 0.30$ \\
\hline Weight (kg) & $61.8 \pm 1.20 *$ & $70.7 \pm 0.40$ & $53.1 \pm 0.80^{*}$ & $57.9 \pm 0.50$ \\
\hline $\mathrm{BMI}\left(\mathrm{kg} / \mathrm{m}^{2}\right)$ & $22.9 \pm 0.41 *$ & $25.1 \pm 0.13$ & $22.7 \pm 0.30 *$ & $23.6 \pm 0.18$ \\
\hline Cholesterol(mmol/L) & $4.87 \pm 0.13$ & $4.91 \pm \pm 0.05$ & $5.08 \pm 0.12 *$ & $5.36 \pm 0.06$ \\
\hline Triglyceride $(\mathrm{mmol} / \mathrm{l})$ & $1.57 \pm 0.10$ & $1.90 \pm 0.07$ & $1.37 \pm 0.09$ & $1.57 \pm 0.06$ \\
\hline $\mathrm{HDL}(\mathrm{mmol} / \mathrm{l})$ & $1.27 \pm 0.04$ & $1.23 \pm 0.02$ & $1.48 \pm 0.04$ & $1.50 \pm 0.02$ \\
\hline $\mathrm{LDL}(\mathrm{mmol} / \mathrm{l})$ & $3.10 \pm 0.12$ & $3.08 \pm 0.04$ & $3.20 \pm 0.12$ & $3.37 \pm 0.05$ \\
\hline $\mathrm{FBG}(\mathrm{mmol} / \mathrm{l})$ & $5.22 \pm 0.14$ & $5.33 \pm 0.05$ & $5.24 \pm 0.09$ & $5.30 \pm 0.06$ \\
\hline $25(\mathrm{OH}) \mathrm{D}(\mathrm{nmol} / \mathrm{l})$ & $45 \pm 2.00$ & $46 \pm 1.00$ & $41.0 \pm 2.00$ & $43.0 \pm 1.00$ \\
\hline $\mathrm{P}(\mathrm{mmol} / \mathrm{L})$ & $0.86 \pm 0.03$ & $0.91 \pm 0.01$ & $1.04 \pm 0.03$ & $1.04 \pm 0.02$ \\
\hline $\mathrm{Ca}(\mathrm{mmol} / \mathrm{l})$ & $2.24 \pm 0.03$ & $2.25 \pm 0.01$ & $2.28 \pm 0.03$ & $2.26 \pm 0.01$ \\
\hline $\mathrm{FN}-\mathrm{BMD}\left(\mathrm{g} / \mathrm{cm}^{2}\right)$ & $0.58 \pm 0.01 *$ & $0.76 \pm 0.01$ & $0.54 \pm 0.01 *$ & $0.69 \pm 0.01$ \\
\hline $\operatorname{LS}-\mathrm{BMD}\left(\mathrm{g} / \mathrm{cm}^{2}\right)$ & $0.81 \pm 0.02 *$ & $0.98 \pm 0.01$ & $0.68 \pm 0.01 *$ & $0.88 \pm 0.01$ \\
\hline TH-BMD $\left(\mathrm{g} / \mathrm{cm}^{2}\right)$ & $0.74 \pm 0.01 *$ & $0.93 \pm 0.01$ & $0.67 \pm 0.01 *$ & $0.83 \pm 0.01$ \\
\hline $\mathrm{FN}-\mathrm{CT}(\mathrm{mm})$ & $0.11 \pm 0.00 *$ & $0.15 \pm 0.00$ & $0.10 \pm 0.00 *$ & $.13 \pm 0.00$ \\
\hline $\mathrm{FN}-\mathrm{SM}\left(\mathrm{cm}^{3}\right)$ & $1.32 \pm 0.03 *$ & $1.69 \pm 0.02$ & $0.94 \pm 0.02 *$ & $1.17 \pm 0.01$ \\
\hline $\mathrm{FN}-\mathrm{CSMI}\left(\mathrm{cm}^{4}\right)$ & $2.39 \pm 0.07 *$ & $3.05 \pm 0.04$ & $1.48 \pm 0.04 *$ & $1.86 \pm 0.03$ \\
\hline $\mathrm{FN}-\mathrm{CSI}\left(\mathrm{g} \cdot \mathrm{kg}^{-1} \cdot \mathrm{m}^{-1}\right)$ & $3.43 \pm 0.08 *$ & $3.90 \pm 0.03$ & $3.23 \pm 0.05^{*}$ & $3.77 \pm 0.04$ \\
\hline $\mathrm{FN}-\mathrm{CSA}\left(\mathrm{cm}^{2}\right)$ & $1.99 \pm 0.03^{*}$ & $2.60 \pm 0.02$ & $1.62 \pm 0.03 *$ & $2.06 \pm 0.01$ \\
\hline FN-BR & $16.6 \pm 0.29 *$ & $12.5 \pm 0.08$ & $15.7 \pm 0.31 *$ & $12.3 \pm 0.12$ \\
\hline MOF (\%) & $4.50 \pm 0.30 *$ & $2.50 \pm 0.10$ & $6.10 \pm 0.30^{*}$ & $3.60 \pm 0.10$ \\
\hline $\mathrm{HF}(\%)$ & $2.60 \pm 0.30 *$ & $0.60 \pm 0.00$ & $2.70 \pm 0.20 *$ & $0.70 \pm 0.00$ \\
\hline TyG index & $8.69 \pm 0.07$ & $8.81 \pm 0.03$ & $8.54 \pm 0.06$ & $8.66 \pm 0.03$ \\
\hline TyG-BMI index & $199.1 \pm 4.27 *$ & $221.7 \pm 1.56$ & $193.8 \pm 2.98^{*}$ & $205.2 \pm 1.89$ \\
\hline Smoke, \%(n) & $46.7(21)$ & $34.8(149)$ & $1.1(4)$ & $0(0)$ \\
\hline Drink, $\%(\mathrm{n})$ & $84.4(38)$ & $18.9(66)$ & $0.4(1)$ & $0(0)$ \\
\hline Previous fracture,(n) & $8.9(4)$ & $6.5(28)$ & $15.3(12)$ & $13(35)$ \\
\hline $\begin{array}{l}\text { Parental hip fracture, } \% \\
\text { (n) }\end{array}$ & $6.7(3) *$ & $21.8(54)$ & $12.5(10)$ & $11.5(32)$ \\
\hline
\end{tabular}

BMI, body mass index; FN: femoral neck; TH:total hip; LS:lumber spine;BMD: body mineral density; CT: cortical thickness; CSMI: Cross sectional moment of inertia; CSI: compression strength index; CSA: Cross sectional area; SM: Section modulus ;BR: buckling ratio ; CT: Cortical 
thickness ; MOF: major osteoporosis fracture; HF: hip fracture; HDL-C high density lipoprotein cholesterol, LDL-C low density lipoprotein cholesterol, FPG fasting plasma glucose; TyG triglyceride glucose index, TyG-BMI combined TyG and BMI.

$*: \mathrm{p}<0.05$

Men
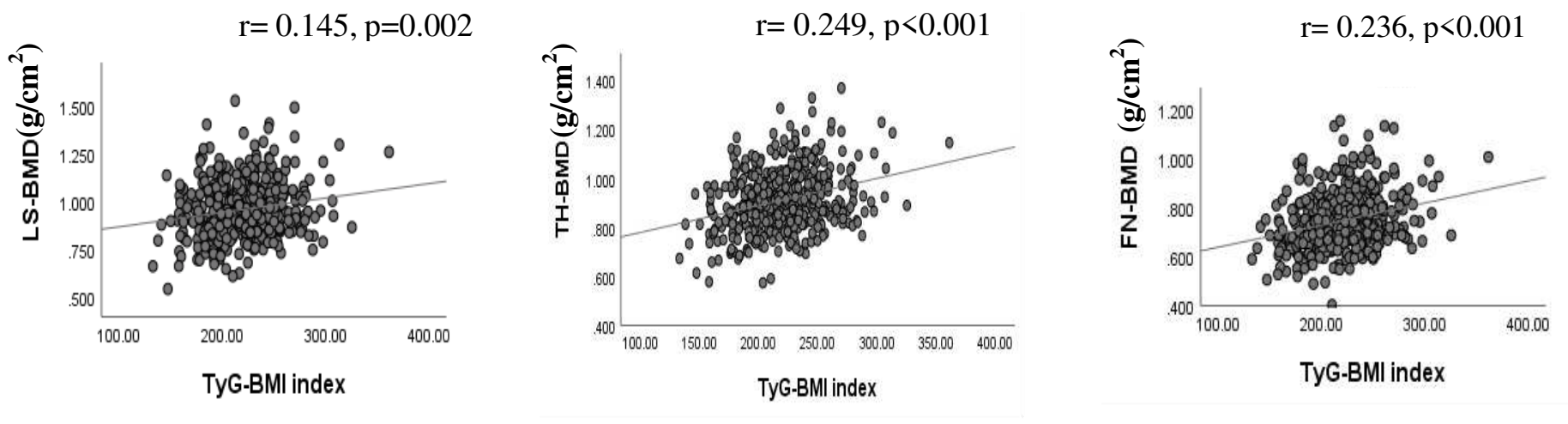

Postmenopausal women
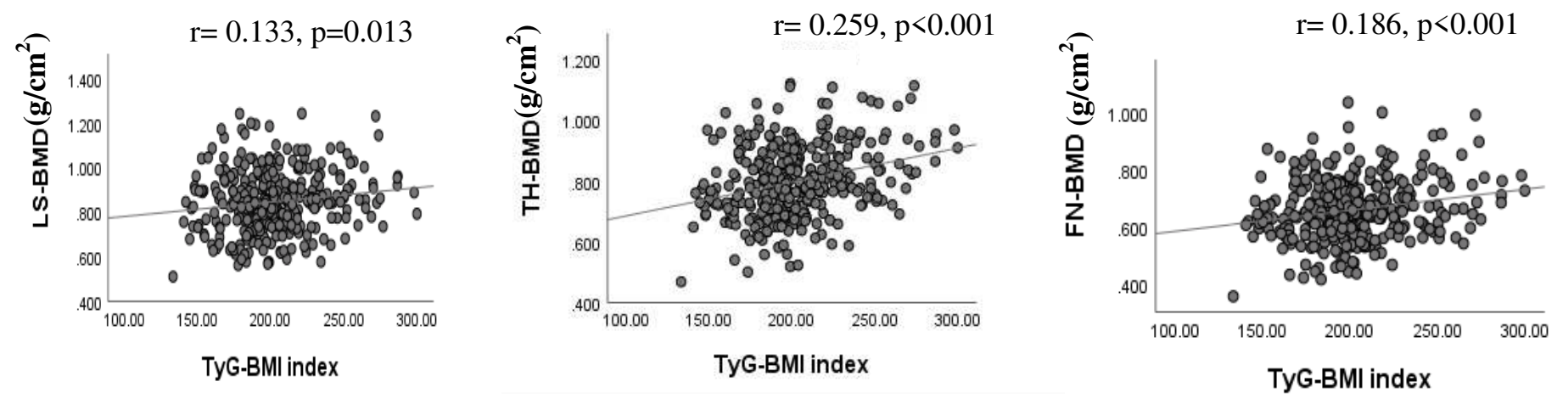

Figure1. Correlations of serum TyG-BMI level with BMD.

FN: femoral neck; TH:total hip; LS:lumber spine;BMD: body mineral density;

TyG triglyceride glucose index, TyG-BMI combined TyG and BMI. 
Table2 Correlations of triglyceride glucose-body mass index and bone strength and risk of fracture

\begin{tabular}{lcc} 
& men & women \\
& $\mathrm{r}$ & $\mathrm{r}$ \\
\hline $\mathrm{FN}-\mathrm{CSMI}\left(\mathrm{cm}^{4}\right)$ & $0.144^{* *}$ & $0.129^{*}$ \\
$\mathrm{FN}-\mathrm{CSI}\left(\mathrm{g} \cdot \mathrm{kg}^{-1} \cdot \mathrm{m}^{-1}\right)$ & $-0.418^{* *}$ & $-0.408^{* *}$ \\
$\mathrm{FN}-\mathrm{CSA}\left(\mathrm{cm}^{2}\right)$ & $0.219^{* *}$ & $0.190^{* *}$ \\
FN-BR & $-0.206^{* *}$ & $-0.119^{*}$ \\
FN-CT $(\mathrm{mm})$ & $0.237^{* *}$ & $0.185^{* *}$ \\
FN-SM $\left(\mathrm{cm}^{3}\right)$ & $0.177^{* *}$ & $0.158^{* *}$ \\
MOF & -0.076 & 0.05 \\
HF & $-0.178^{* *}$ & -0.064 \\
\hline
\end{tabular}

FN: femoral neck; CT: cortical thickness; CSMI: Cross sectional moment of inertia; CSI: compression strength index; CSA: Cross sectional area; SM: Section modulus ;BR: buckling ratio; TyG-BMI combined TyG and BMI; MOF:major osteoporotic fracture;HF:hip fracture 
Table3a Lineal regression analysis between the TyG-BMI index and the densitometry parameters.

\begin{tabular}{|c|c|c|c|c|}
\hline \multicolumn{5}{|c|}{ men } \\
\hline & unadjusted & Model1 & Model2 & Model3 \\
\hline & & $\beta$ & $\beta$ & $\beta$ \\
\hline $\mathrm{FN}-\mathrm{CSI}\left(\mathrm{g} \cdot \mathrm{kg}^{-1} \cdot \mathrm{m}^{-1}\right)$ & $-0.401 * *$ & $-0.414 * *$ & $-0.426 * *$ & $-0.426 * *$ \\
\hline $\mathrm{FN}-\mathrm{CSMI}\left(\mathrm{cm}^{4}\right)$ & $0.213 * *$ & $0.191 * *$ & $0.182 * *$ & $0.182 * *$ \\
\hline $\mathrm{FN}-\mathrm{SM}\left(\mathrm{cm}^{3}\right)$ & $0.238 * *$ & $0.212 * *$ & $0.205 * *$ & $0.204 * *$ \\
\hline $\mathrm{FN}-\mathrm{CT}(\mathrm{mm})$ & $0.263 * *$ & $0.238 * *$ & $0.230 * *$ & $0.231 * *$ \\
\hline FN-BR & $-0.227 * *$ & $-0.202 * *$ & $-0.194 * *$ & $-0.195 * *$ \\
\hline $\mathrm{FN}-\mathrm{CSA}\left(\mathrm{cm}^{2}\right)$ & $0.2693 * *$ & $0.238 * *$ & $0.229 * *$ & $0.229 * *$ \\
\hline $\mathrm{FN}-\mathrm{BMD}\left(\mathrm{g} / \mathrm{cm}^{2}\right)$ & $0.265^{* *}$ & $0.234 * *$ & $0.223 * *$ & $0.224 * *$ \\
\hline $\mathrm{LS}-\mathrm{BMD}\left(\mathrm{g} / \mathrm{cm}^{2}\right)$ & $0.173 * *$ & $0.176^{* *}$ & $0.185 * *$ & $0.185 * *$ \\
\hline TH-BMD $\left(\mathrm{g} / \mathrm{cm}^{2}\right)$ & $0.293 * *$ & $0.275^{* *}$ & $0.27 * *$ & $0.271 * *$ \\
\hline MOF & -0.067 & -0.031 & -0.055 & -0.108 \\
\hline $\mathrm{HF}$ & -0.125 & $-0.078 *$ & $-0.116^{*}$ & $-0.141 *$ \\
\hline
\end{tabular}

Model 1 adjusted age; model2 :adjusted age, smoke, drink; model3 :adjusted age, smoke, drink, previous fracture, parental hip fracture.

FN: femoral neck; TH: total hip; LS:lumber spine;BMD: body mineral density; CSI: compression strength index; CSMI: Cross sectional moment of inertia ; SM: Section modulus ; CT: Cortical thickness ; BR: buckling ratio ; CSA: Cross sectional area; MOF:major osteoporotic fracture;HF:hip fracture; TyG triglyceride glucose index, TyG-BMI combined TyG and BMI, $* *: \mathrm{p}<0.001 ; *: \mathrm{p}<0.05$. 
Table3b Lineal regression analysis between the TyG-BMI index and the densitometry parameters.

\begin{tabular}{|c|c|c|c|c|}
\hline \multicolumn{5}{|c|}{ women } \\
\hline & unadjusted & Model1 & Model2 & Model3 \\
\hline & & $\beta$ & $\beta$ & $\beta$ \\
\hline $\mathrm{FN}-\mathrm{CSI}\left(\mathrm{g} \cdot \mathrm{kg}^{-1} \cdot \mathrm{m}^{-1}\right)$ & $-0.409 * *$ & $-0.367 * *$ & $-0.367 * *$ & $-0 / 368 * *$ \\
\hline $\mathrm{FN}-\mathrm{CSMI}\left(\mathrm{cm}^{4}\right)$ & $0.147^{*}$ & $0.194 * *$ & $0.194 * *$ & $0.194 * *$ \\
\hline $\mathrm{FN}-\mathrm{SM}\left(\mathrm{cm}^{3}\right)$ & $0.180 * *$ & $0.228 * *$ & $0.228 * *$ & $0.227 * *$ \\
\hline $\mathrm{FN}-\mathrm{CT}(\mathrm{mm})$ & $0.213 * *$ & $0.342 * *$ & $0.341 * *$ & $0.342 * *$ \\
\hline FN-BR & $-0.167 * *$ & $0.283 * *$ & $0.283 * *$ & $0.282 * *$ \\
\hline $\mathrm{FN}-\mathrm{CSA}\left(\mathrm{cm}^{2}\right)$ & $0.2203^{* *}$ & $0.283 * *$ & $0.283 * *$ & $0.282 * *$ \\
\hline $\mathrm{FN}-\mathrm{BMD}\left(\mathrm{g} / \mathrm{cm}^{2}\right)$ & $0.214 * *$ & $0.279 * *$ & $0.279 * *$ & $0.279 * *$ \\
\hline $\operatorname{LS}-\mathrm{BMD}\left(\mathrm{g} / \mathrm{cm}^{2}\right)$ & $0.142 * *$ & $0.192 * *$ & $0.193 * *$ & $0.192 * *$ \\
\hline TH-BMD $\left(\mathrm{g} / \mathrm{cm}^{2}\right)$ & $0.292 * *$ & $0.411 * *$ & $0.41 * *$ & $0.415 * *$ \\
\hline MOF & 0.015 & $-0.148 *$ & $-0.148 *$ & $-0.219 *$ \\
\hline-0.089 & & $-0.293 * *$ & $-0.293^{* *}$ & $-0.309 * *$ \\
\hline
\end{tabular}

Model 1 adjusted age; model2 :adjusted age, smoke, drink; model3 :adjusted age, smoke, drink, previous fracture, parental hip fracture.

FN: femoral neck; TH: total hip; LS:lumber spine;BMD: body mineral density; CSI: compression strength index; CSMI: Cross sectional moment of inertia ; SM: Section modulus ; CT: Cortical thickness ; BR: buckling ratio ; CSA: Cross sectional area; MOF:major osteoporotic fracture;HF:hip fracture;TyG triglyceride glucose index, TyG-BMI combined TyG and BMI,

$* *$ : $\mathrm{p}<0.001 ; * \mathrm{p}<0.05$ 


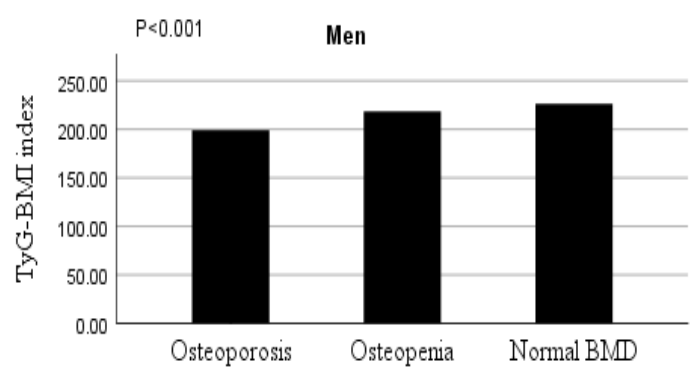

a

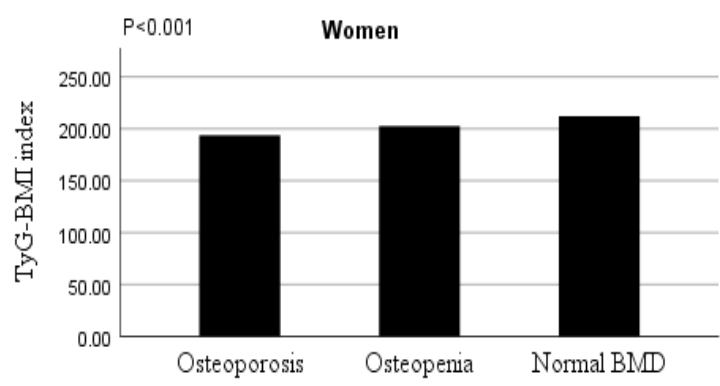

b

Figure2 Distributions of the TyG-BMI index according to the bone health status. 
Table4 Multivariable logistic regression analyses between possible predictors and osteoporosis

\begin{tabular}{lcccc}
\hline & Unadjusted OR (95\% CI) & P-value & Adjusted OR (95\% CI) & $\begin{array}{c}\text { P- } \\
\text { value }\end{array}$ \\
\hline Age & $0.929(0.907,0.950)$ & $<0.001$ & $0.919(0.892,0.947)$ & $<0.001$ \\
Sex, female & $0.393(0.263,0.587)$ & $<0.001$ & $0.486(0.266,0.889)$ & 0.019 \\
History of Parental hip fracture & $0.823(0.434,1.559)$ & 0.550 & $0.872(0.414,1.836)$ & 0.719 \\
Current Smoker & $0.784(0.473,1.730)$ & 0.346 & $0.353(0.176,0.709)$ & 0.003 \\
Current Drinker & $0.389(0.176,0.860)$ & 0.020 & $1.588(0.608,4.145)$ & 0.345 \\
25(OH)D & $1.011(0.996,1.027)$ & 0.142 & $1.018(1.002,1.034)$ & 0.026 \\
TyG-BMI index & $1.022(1.014,1.029)$ & $<0.001$ & $1.019(1.010,1.028)$ & $<0.001$ \\
\hline
\end{tabular}

Adjusted with age, sex, 25(OH)D, current smoker, current drinker. 


\section{Supplementary Files}

This is a list of supplementary files associated with this preprint. Click to download.

- supplemental1.docx 